domain and a third of them had delays in 2 or more domains on the ASQ-3 assessment. Based on ASQ: SE, 33\% of these children were reported to have mild to significant social-emotional developmental delays. $48 \%$ of the children were reported with behavioral and emotional concerns in CBCL assessments. $30 \%$ of the children had incomplete vaccination. A third of caregivers had mental wellness concerns as indicated by PHQ-9 and GAD-7 scores. M-CHAT and PICCOLO results are being analysed and will be presented in the conference.

Conclusions Anchor programme and its holistic evaluation showed that children with ACES and their families are a vulnerable group with high needs. It is imperative that these needs are identified in a timely manner to ensure that targeted support services are provided through home visitation and inter-agency collaboration.

\section{EFFICACY OF WEARABLE PHYSICAL ACTIVITY TRACKERS, FUNCTION AND PARTICIPATION MEASURES IN CHILDREN WITH CEREBRAL PALSY; REVIEW OF THE EVIDENCE}

Natalie R Walsh, Hani F Ayyash. UK

\subsection{6/bmjpo-2021-RCPCH.240}

Background Cerebral Palsy is the primary cause of early physical disability in children and affects approximately 110,000 people, with a prevalence of 2-2.5 per 1000 live births in the United Kingdom. Young people with Cerebral Palsy have reduced physical fitness with an estimated, maximum aerobic capacity of $15-28 \%$ less than healthy peers. This may lead to health consequences, such as hypertension and obesity.

The UK physical activity guidelines for healthy children (5-18 years), advise 60-minutes of daily moderate-intensityphysical-activity (MIPA). However, these guidelines do not include children with long-term conditions such as Cerebral Palsy. Additionally, appropriate outcome measures are required to record the effects of interventions on physical activity, functional-mobility, and participation in children with disabilities.

Objectives To review the evidence for the use of the physical activity, functional-mobility, and participation measures for young people with Cerebral Palsy.

Methods The current research was screened using the key search terms Cerebral Palsy, exercise, and physical activity, to critique the literature for physical activity, functional mobility, and participation measures for young people with Cerebral Palsy. These included wearable physical activity trackers (WPAT), the Physical Activity Questionnaire for Older Children (PAQ-C), the Timed-up-and-Go (TUG) and the Child and Adolescent Scale of Participation (CASP).

Results Physical activity can be measured subjectively or objectively. WPAT can be used to track objective PA in children with chronic diseases to record health-outcomes. Furthermore, a benchmark of 12,000-16,000 steps-a-day, has been suggested to meet the recommended daily PA (60-minutes). However, the articles indicate limitations in the variability of devices, placement, duration, activity, and environment.

The PAQ-C papers demonstrated some evidence for acceptable psychometric properties and provided normative scores for recording PA levels. However, the impact of different seasons, school holidays, and recruiting healthy children samples, needs to be considered to record subjective PA levels, in children with disabilities.

The TUG has been used to measure balance, postural control, mobility, and function in children with CP. The literature suggests high reliability and minimal detectable change and minimal clinical important difference values of the TUG in children. However, the small sizes used this research must be acknowledged.

The research reviewed for the CASP were predominantly used in TBI and arm-injuries and suggested some preliminary evidence for the psychometric properties in children with neurological conditions.

Conclusions The literature review indicates preliminary evidence for the use of WPAT, PAQ-C, TUG, and CASP to record the effects of interventions in children with disabilities, such as Cerebral Palsy. However, future research should focus on the feasibility, and applicability, of public health guidelines for physical activity, intended for children with CP, to improve and maintain health and well-being.

\section{X-LINKED ICHTHYOSIS, AN UNDER DIAGNOSED GENETIC SKIN DISORDER IN CHILDREN-CASE REPORT}

Smitha Bhaskaran, Pramod Nair. UK

\subsection{6/bmjpo-2021-RCPCH.241}

Background X-Linked Ichthyosis is the second most common cause of ichthyosis in children.It is due to deficiency of steroid sulfatase(STS) either due to deletion or mutation of the STS gene at Chromosome Xp22.3.Considering this condition in severe cases of eczema is important in patient management and genetic counselling.

Objectives

Methods We report a male infant born to non-consanguineous parents who had dry skin since birth.He was born at $40+1$ weeks of gestation by emergency caesarean section due to variation in foetal heart rate.He had a stormy beginning to life, needing full resuscitation at birth and 6 days of ECMO for severe PPHN.He was noted to have dry scaly skin since which got worsened over time and was refractory to treatment.A genetic study was sent as part of the initial workup which later showed micro deletion at chromosome Xp22.31 commonly associated with STS deficiency.This result correlated with his clinical condition and a genetic referral was made for carrier state detection and counselling.

Results

Conclusions X-linked recessive Ichthyosis is exclusively manifested in males and females are asymptomatic carriers.STS gene located at chromosome $\mathrm{Xp} 22.3$ is defective either by deletion or point mutation resulting in deficiency of steroid sulfatase which normally keeps the skin moisturised.Deficient STS results in accumulation of cholesterol sulfate in stratum corneum of skin leading to ichthyosis. There can be associated corneal opacities and rarely cryptorchidism. The deficiency of enzyme can result in reduced production of maternal estriol during late pregnancy, which may affect induction of labour and delivery. We do not know whether his genetic condition had any contribution to is stormy neonatal period and need for ECMO.It would be a useful research if find similar associations in other children with $\mathrm{X}$-linked Ichthyosis. However it is a very rare condition and should be considered in the differential diagnosis of dry 